\title{
Performance of the ALIBAVA portable readout system with irradiated and non-irradiated microstrip silicon sensors
}

\author{
Ricardo Marco-Hernández ${ }^{1}$ \\ Instituto de Física Corpuscular (CSIC-UV) \\ Edificio Institutos de Investigación. Poligono de la Coma, s/n. E-46980 Paterna(Valencia), Spain \\ E-mail: rmarcoeific.uv.es
}

\section{On behalf of the ALIBAVA Collaboration}

\begin{abstract}
A readout system for microstrip silicon sensors has been developed as a result of collaboration among the University of Liverpool, the CNM of Barcelona and the IFIC of Valencia. The name of this collaboration is ALIBAVA and it is integrated in the RD50 Collaboration. This system is able to measure the collected charge in one or two microstrip silicon sensors by reading out all the channels of the sensor(s), up to 256, as an analogue measurement. The system uses two Beetle chips to read out the detector(s). The Beetle chip is an analogue pipelined readout chip used in the $\mathrm{LHCb}$ experiment. The system can operate either with non-irradiated and irradiated sensors as well as with n-type and p-type microstrip silicon sensors. Heavily irradiated sensors will be used at the SLHC, so this system is being used to research the performance of microstrip silicon sensors in conditions as similar as possible to the SLHC operating conditions The system has two main parts: a hardware part and a software part. The hardware part acquires the sensor signals either from external trigger inputs, in case of a radioactive source setup is used, or from a synchronised trigger output generated by the system, if a laser setup is used. The software controls the system and processes the data acquired from the sensors in order to store it in an adequate format. The main characteristics of the system will be described. Results of measurements acquired with n-type and p-type irradiated and non-irradiated detectors using both the laser and the radioactive source setup will be also presented and discussed.
\end{abstract}

European Physical Society Europhysics Conference on High Energy Physics EPS-HEP 2009,

Krakow, Poland

July 16-22, 2009

\footnotetext{
$1 \quad$ Speaker
} 


\section{Introduction}

The main properties of highly irradiated microstrip silicon sensors must be studied since a high luminosity is intended to be achieved at SLHC (Super Large Hadron Collider) experiments [1]. Particularly, the charge collected when a charged particle crosses the detector is important for the detector performance. It would be interesting to test this kind of detectors with an electronic system as similar as possible to those used at the LHC (Large Hadron Collider) experiments, so a front-end readout chip [2] as those used at the LHC experiments should be used. Furthermore, an analogue measurement of the front-end pulse shape is preferred over a binary one for charge collection research.

Therefore, an electronic system which can acquire an analogue measurement has been developed. The system can be used with a laser setup, where a laser light is generated by exciting a laser source with a pulsed signal. It can be used also with a radioactive source setup, where the charged particles are generated randomly. The aim is reconstructing the analogue pulse shape at the readout chip front-end with the highest fidelity.

The system is compact and portable. It has its own supply system and contains two Beetle readout chips [2] to acquire the detector signals. It is connected via USB (Universal Serial bus) to a PC host, which stores and processes the data acquired. The user controls the system with the PC software in communication with a FPGA (Field programmable Gate Array) which interprets and executes the commands. The system can be used with two different laboratory setups so it has an external trigger input, from one or two photomultipliers (radioactive source), and it generates a trigger output for pulsing an external excitation source (laser setup).

\section{System architecture}

The system has two main parts: a hardware part and a software part (Figure 1). The hardware part acquires the sensor signals either from external trigger inputs, in case of a radioactive source setup is used, or from a synchronised trigger output generated by the system, if a laser setup is used. The hardware is made of a daughter board and a mother board. The daughter board contains two Beetle readout chips as well as the circuitry necessary to connect the Beetle chips with the motherboard. Fan-ins and a detector board are used to interface the sensors.

The Beetle chip has 128 channels, each consisting of a low-noise charge-sensitive preamplifier, an active CR-RC pulse shaper and a buffer. Each channel is sampled with a $40 \mathrm{MHz}$ frequency into an analogue pipeline. The signal stored in the pipeline is serialized and two current drivers bring the serialized data off chip in 3600 ns. For test and calibration purposes a charge injector with adjustable pulse height is implemented on each channel. The bias settings and various other parameters like the trigger latency can be controlled via a standard $\mathrm{I}^{2} \mathrm{C}$-interface. All digital control and data signals, except those for the $\mathrm{I}^{2} \mathrm{C}$-ports, are routed via LVDS ports.

The mother board is intended to process and digitize the analogue data that come from the analogue readout chips. It also processes the trigger input signal in case of radioactive source 
setup or it generates an output trigger signal if a laser setup is used. Moreover, it controls the whole system and it communicates with the PC software via USB, using a FPGA with an embedded processor and custom logic. The software controls the whole system and processes the data acquired by the detectors in order to store it in an adequate format. This format is compatible with software used for further data analysis, in the ROOT framework. With this software the system can be configured and calibrated. Acquisitions with a laser setup or a radioactive source setup can be carried out as well.

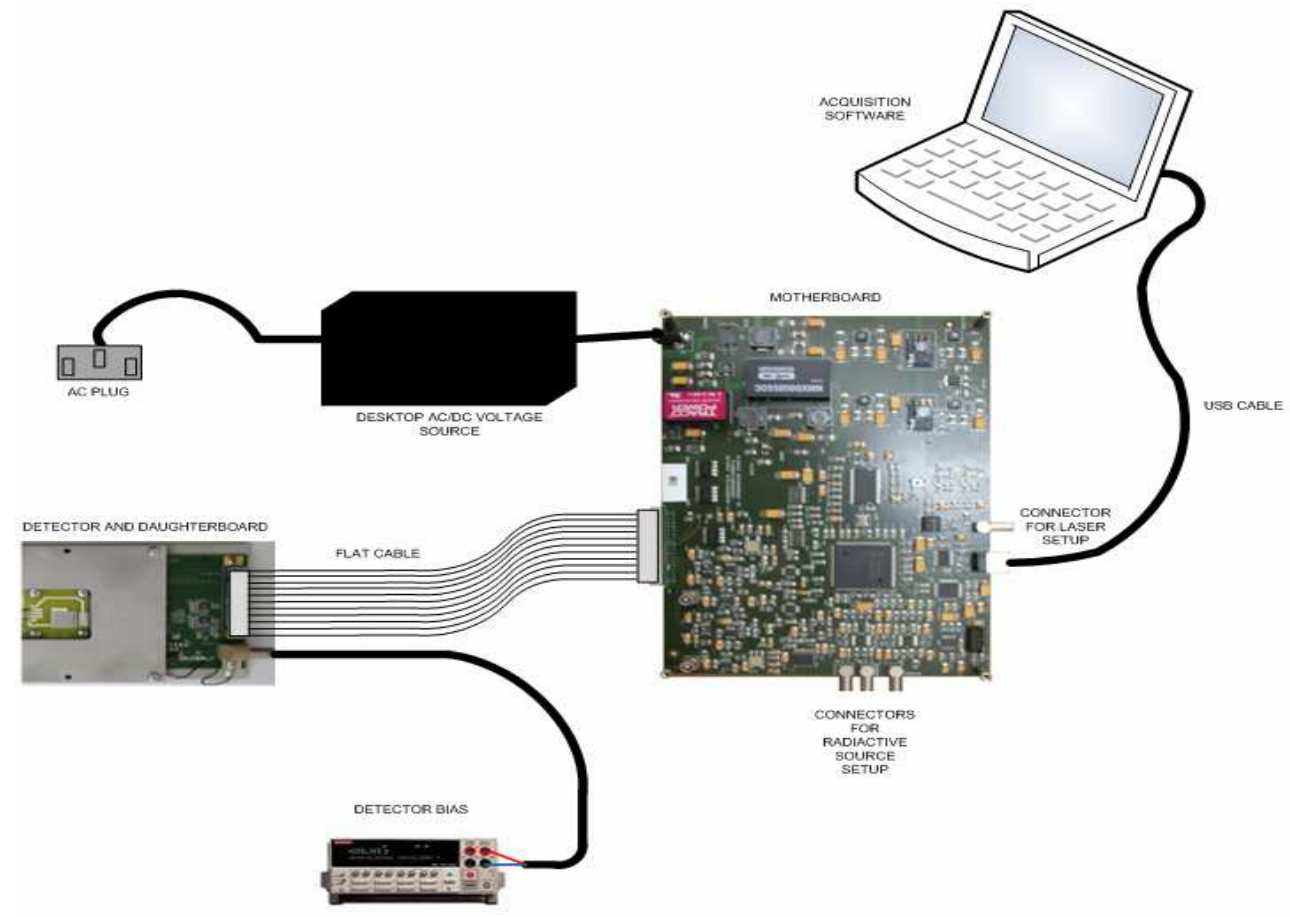

Figure 1. Main parts of the readout system

The main reason for dividing the hardware into two boards is to preserve the rest of the hardware from the extreme environment (radiation or very low temperatures) where the detectors operate. Analogue data signals coming from the daughter board, digital control signals to the Beetle chips as well as the power supply for the daughter board circuitry run across a twisted flat cable. The detector's high voltage is provided by an external power supply.

\section{Measurements}

Measurements acquired with n-type and p-type irradiated and non-irradiated sensors using both the laser and the radioactive source setups have been carried out with the system. The system is able to reconstruct the pulse shape of the Beetle chip shaper output both for a laser setup and a source setup as well as calibration data. Therefore, the collected charge can be obtained from these measurements both for irradiated and non-irradiated detectors.

A plot representing the collected charge versus the depletion voltage of the detector for ATLAS07 irradiated and non-irradiated detectors is shown in Figure 2. In this figure, the different curves correspond to sensors with different irradiation doses (irradiation with neutrons) and these curves have been obtained with different readout systems. VLC denotes data carried 
out in Valencia with the ALIBAVA system. LIV and LJUB denotes data carried out in Liverpool and Ljubljana with other readout systems and the same type of sensors for comparision.

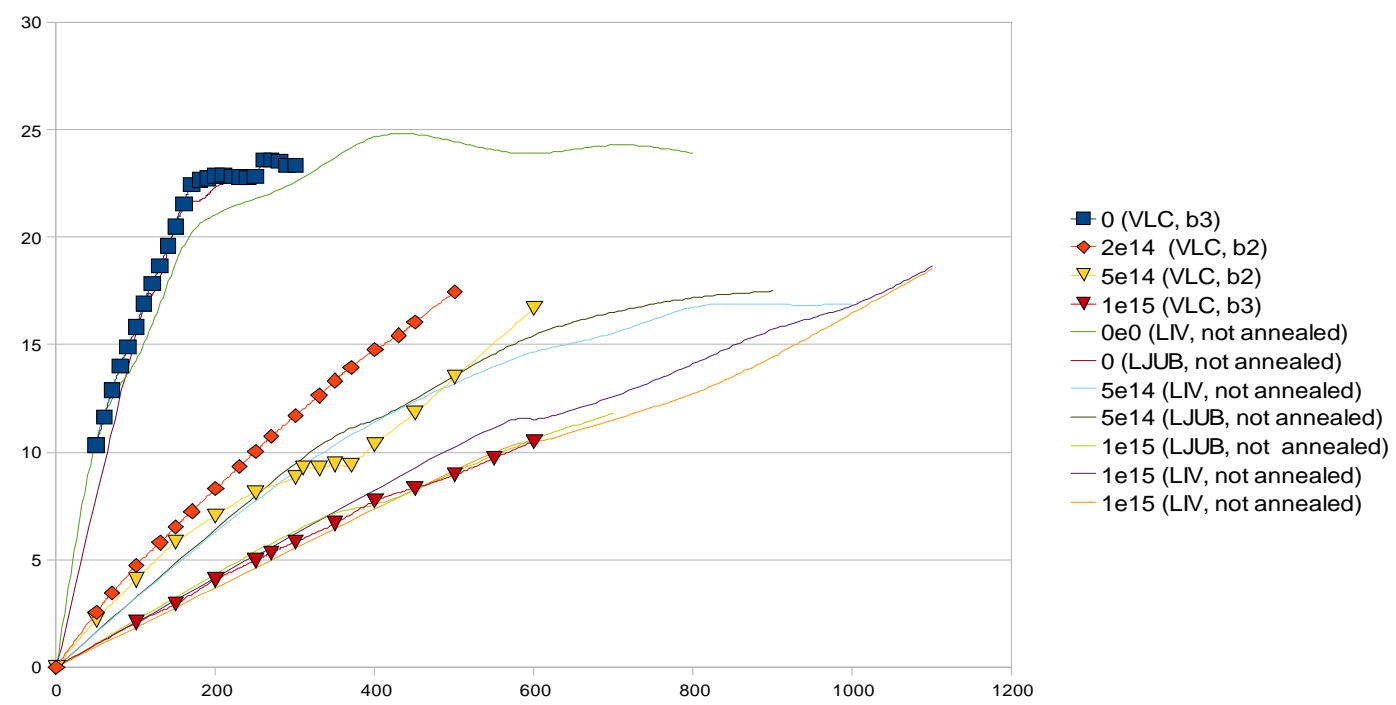

Figure 2. Measurements of collected charge versus depletion voltage for ATLAS07 sensors irradiated at diferent doses and mesured with different systems. VLC stands for measurements carried out with the ALIBAVA system in Valencia, b2 and b3 refers to different daughter boards. LIV and LJUB stands for measurements carried out in Liverpool and Ljubljana with other readout systems [2].

ATLAS07 sensors are n-on-p FZ microstrip sensors of $1 \mathrm{~cm}^{2}, 80 \mu \mathrm{m}$ of pitch and $300 \mu \mathrm{m}$ of thickness. Measurements with n-on-n detectors have been reported in [4]. Charge collection data has been obtained by a full scan for the represented Vbias with a near infrared laser (1064 $\mathrm{nm}$ ) Measurements with a $90 \mathrm{Sr}$ source (low activity) for a few Vbias have been carried out to calibrate the laser measurements.

For non-irradiated detectors the SNR of the system with a $\beta$ source is 22 . With the laser setup, the signal is much larger, so there is no concern about the SNR. The SNR in irradiated detectors should be lower since the noise increases because of the radiation effects and the reduced collected charge. However, since the measurements can be acquired with the detectors cooled (i.e. the daughterboard) and the radiation effects on the collected charge can be partially counteracted by increasing the depletion voltage of the detector, the noise can be minimized.

\section{Conclusions}

The design of a readout system for microstrip silicon sensors has been reported. This system has been developed in the framework of the ALIBAVA collaboration (University of Liverpool, CNM of Barcelona and IFIC of Valencia) which is integrated in the RD50 collaboration. The system can operate with different types (p-type and n-type) and different 
sizes (up to $3 \mathrm{~cm}^{2}$ ) of microstrip silicon sensors. The system has up to 256 input channels and it has been designed to operate with both irradiated and non-irradiated sensors. The hardware and software used in order to design the system are generally applicable to microstrip silicon sensors. Two different laboratory setups (laser setup or radioactive source setup) can be used with the system, which is useful for comparing results with the same detector. The system has been tested with these two laboratory setups and works correctly.

\section{References}

[1] F. Ruggiero et al., LHC Luminosity and Energy Upgrade: A Feasibility Study. LHC Project Report 626, 2002.

[2] S. Löchner and M. Schmelling, The Beetle reference manual for Beetle version 1.3/1.4/1.5, LHCb note 2005-105, August 22, 2006.

[3] C. Lacasta, Sensor measurements at IFIC, ATLAS Tracker Upgrade Workshop, NIKHEF, Holland, November 2008.

[4] U.Soldevila et al., Neutron irradiation for p-type sensors.Detector characterization with ALIBAVA system, 14th RD50 Workshop, Freiburg, Germany, June 2009. 